\title{
Safety and efficacy of montelukast as adjunctive therapy for treatment of asthma in elderly patients
}

This article was published in the following Dove Press journal:

Clinical Interventions in Aging

30 September 2013

Number of times this article has been viewed

\section{Nicola Scichilone \\ Salvatore Battaglia \\ Alida Benfante \\ Vincenzo Bellia}

Dipartimento Biomedico di Medicina Interna e Specialistica, University of Palermo, Palermo, Italy
Correspondence: Nicola Scichilone Dipartimento Biomedico di Medicina Interna e Specialistica, University of Palermo, Palermo, Italy, via Trabucco I80, 90। 46 Palermo, Italy

Tel +3909 16802655

Fax +3909 16882842

Email nicola.scichilone@unipa.it
Abstract: Asthma is a disease of all ages. This assumption has been challenged in the past, because of several cultural and scientific biases. A large body of evidence has accumulated in recent years to confirm that the prevalence of asthma in the most advanced ages is similar to that in younger ages. Asthma in the elderly may show similar functional and clinical characteristics to that occurring in young adults, although the frequent coexistence of comorbid conditions in older patients, together with age-associated changes in the human lung, may lead to more severe forms of the disease. Management of asthma in the elderly follows specific guidelines that apply to all ages, although most behaviors are pure extrapolation of what has been tested in young ages. In fact, age has always represented an exclusion criterion for eligibility to clinical trials. This review focuses specifically on the safety and efficacy of leukotriene modifiers, which represent a valid option in the treatment of allergic asthma, both as an alternative to first-line drugs and as add-on treatment to inhaled corticosteroids. Available studies specifically addressing the role of montelukast in the elderly are scarce; however, leukotriene modifiers have been demonstrated to be safe in this age group, even though cases of acute hepatitis and occurrence of Churg-Strauss syndrome have been described in elderly patients; whether this is associated with age is to be confirmed. Furthermore, leukotriene modifiers provide additional benefit when added to regular maintenance therapy, not differently from young asthmatics. In elderly patients, the simpler route of administration of leukotriene modifiers, compared with the inhaled agents, could represent a more effective strategy in improving the outcomes of asthma therapy, given that unintentional nonadherence with inhalation therapy represents a complex problem that may lead to significant impairment of asthma symptom control.

Keywords: leukotriene antagonists, asthma, treatment, airway inflammation, aging

\section{Introduction}

Bronchial asthma is one of the most common chronic diseases worldwide, and by definition is not expected to recover with aging. However, the concept that asthma can affect older individuals has been largely denied in the past, leading to underdiagnosis and/or misdiagnosis and, consequently, undertreatment or inappropriate treatment. The heterogeneity of the clinical and functional presentation of geriatric asthma, including the partial loss of reversibility and of the allergic component, contributes to this misconception. Aging induces structural changes in the airway wall that contribute to the phenomenon of remodeling, and is associated with modifications of the immune system, defined as immunosenescence, which can influence the atopic state in the geriatric age group. Initial observations concerning atopy in the geriatric population, derived from longitudinal studies in the general population, led to the notion that the 
prevalence of atopy decreases with aging, reinforcing the concept that allergic asthma is a rare nosologic entity in the elderly. Today, asthma in the elderly is a well recognized condition, with unique features reflecting the coexistence of other diseases, use of multiple respiratory and nonrespiratory drugs, individual responsiveness to treatment, and differences in the perception of symptoms. The prevalence of asthma in older adults has been demonstrated to be not different from that of younger adults, ranging from $6 \%$ to $10 \% .{ }^{1}$ The burden of senile asthma should be weighed in the perspective of the progressive aging of the population. ${ }^{2}$ In addition, the senescence-associated limitations in functioning and in local and systemic defenses, along with long-term exposure to noxious environmental stimuli, expose the elderly asthmatic to the risk of more frequent and more intense attacks. Taken together, these observations allow us to predict that asthma in the elderly will have a markedly increased impact on public health in the upcoming years. In this context, Bellia et al demonstrated that asthma in the elderly was associated with a higher mortality rate. ${ }^{3}$

In the current review, we describe the contribution of the leukotriene antagonists (LTRAs) in the management of "geriatric" asthma, focusing on the safety and efficacy profiles of the drugs available. To this end, studies specifically addressing the role of leukotriene modifiers in the most advanced age groups are analyzed.

\section{Asthma in the elderly}

The "elderly" are commonly identified as subjects 65 years of age and over; however, for the purposes of the present review, we decided to incorporate studies that refer to populations of different ages, such as 50 years and over, 60 years and over, or 80 years and over. Asthma in the elderly can be distinguished in terms of early or late onset. This identifies two patterns that may have some clinical and functional differences; in particular, "late" identifies those patients who develop asthma at age older than 65 years. ${ }^{4}$ As discussed below, atopy seems more frequent in patients with early-onset asthma than in those with late-onset asthma. Late-onset asthma appears to be a peculiar condition, ${ }^{4}$ perhaps linked with age-associated loss of beta- 2 receptors. Older asthmatics seem to complain less about their symptoms, and Connolly et $\mathrm{al}^{5}$ demonstrated that elderly patients were less aware of experimentally induced bronchoconstriction than younger controls. This observation was confirmed by other studies, ${ }^{6,7}$ supporting the notion that elderly asthmatics can be considered "poor perceivers" of their symptoms. From a clinical perspective, asthma maintains its clinical characteristics in the elderly, although some overlap with features of chronic obstructive pulmonary disease may occur as the patient ages. It should be noted that age per se may lead to cognitive impairment and mood changes, such as anxiety and depression, which may have considerable impact on the occurrence of symptoms. The functional condition of reversible airflow limitation may not be applicable in elderly asthmatics, due to airway remodeling changes that modify the characteristics of airway obstruction towards nonreversible features.

\section{Allergic component}

Allergies have been traditionally considered to be conditions affecting children and young adults. The immune system undergoes an involutional process with a consequent decline in production of immunoglobulins, including IgE. This has led us to consider asthma in the elderly as a disease of nonallergic pathogenesis. As a consequence, atopy has never been taken into consideration in the clinical assessment and management of the geriatric respiratory patient. The decrease in prevalence of allergen sensitization with increasing age e $^{8,9}$ has been interpreted to mean that allergic patients tend to outgrow their allergy as they get older. Although the prevalence of atopy is lower in the elderly compared with the younger population, the clinical significance of this condition and its impact on the comprehensive management of asthma in this age group is still a matter of debate. It is commonly accepted that allergic reactions may have the same or even worse manifestations in the elderly than in younger individuals; however, due to the aging decline in function of various systems, such as the cardiovascular system, older patients may not be able to compensate for the consequences of an allergic reaction. In a recent review of atopy in elderly asthmatics, ${ }^{10}$ we concluded that the prevalence of allergic sensitization in the elderly population with respiratory symptoms is substantial enough to warrant assessment of atopy and the evaluation of the atopic condition. In particular, we suggested that assessment of the allergic condition in this age group could still contribute to improved diagnosis and specific treatment.

\section{Airway inflammation}

Airway inflammation in asthma is a multicellular process involving mainly eosinophils, CD4 T-lymphocytes, and mast cells. Age-related changes in eosinophil function and their potential implications for asthma were investigated in a study by Mathur et $\mathrm{al}^{11}$ showing that peripheral blood eosinophil degranulation in response to interleukin-5 stimulation was significantly decreased in the older age group, whereas eosinophil adhesion, eosinophil chemotaxis, lung function, 
and the percentage of sputum eosinophils were similar in young and older adult asthmatics. The clinical and functional similarities with chronic obstructive pulmonary disease do not necessary imply that the two pathologic conditions also share similar inflammatory patterns. In this regard, Fabbri et $\mathrm{l}^{12}$ clearly demonstrated that asthma in the elderly has a pathology which is markedly different from that of chronic obstructive pulmonary disease, even in the presence of irreversible airway obstruction. Indeed, persistent airflow limitation can develop in nonsmoking patients with asthma and is associated with older age, a longer duration of asthma, sputum eosinophilia, and airway hyperresponsiveness. ${ }^{13}$ Fabbri et $\mathrm{al}^{12}$ showed that asthmatic airway inflammation does not lose its cytologic and histochemical peculiarities with the development of fixed airflow obstruction. These findings were confirmed by another study addressing the inflammatory characteristics of the airways in elderly patients with asthma or chronic obstructive pulmonary disease not differing for lung function. ${ }^{14}$ Taken together, these observations lead us to assume that the inflammatory cascade characterizing the processes occurring in the asthmatics airways is not profoundly affected by age. In the sequence of inflammatory events, several mediators are involved and, among them, a specific role is attributed to the leukotrienes, which are produced by a number of cell types, particularly mast cells, eosinophils, basophils, macrophages, and monocytes. Soon after their characterization, the leukotrienes were described as being implicated in the pathogenesis of asthma, thus becoming targets for therapeutic modulation.

\section{Role of leukotrienes in the inflammatory cascade}

Leukotrienes derive from arachidonic acid, a component of the lipid bilayer of the cell and nuclear membranes. The cytosolic enzyme, phospholipase A2, cleaves arachidonic acid from the lipid bilayer, generating free arachidonic acid. The 5-lipoxygenase enzyme acts upon free arachidonic acid, generating leukotriene $\mathrm{A}_{4}$. Leukotriene $\mathrm{A}_{4}$ is then converted to leukotriene $\mathrm{C}_{4}$ by its synthase, and is then transported into the extracellular microenvironment, where it is converted to leukotriene $\mathrm{D}_{4}$. Finally, this is converted to leukotriene $\mathrm{E}_{4}$. Leukotriene $\mathrm{C}_{4}$, leukotriene $\mathrm{D}_{4}$, and leukotriene $\mathrm{E}_{4}$ all contain cysteine, and are therefore collectively known as the cysteinyl leukotrienes. There are two known receptors to which cysteinyl leukotrienes bind: CysLT-1, which binds to all three cysteinyl leukotrienes and is found on eosinophils, monocytes, airway smooth muscle cells, neutrophils, B cells, plasma cells, and tissue macrophages, and CysLT-2, which has higher affinity for leukotriene $\mathrm{C}_{4}$ and leukotriene $\mathrm{D}_{4}$, and is detected in many tissues of the body, including the cardiovascular system, adrenal glands, the nasal epithelium, and mucus glands. ${ }^{15}$ There is evidence for the presence of cysteinyl leukotriene receptors (CysLT1 and CysLT2) in the human lung; furthermore, Back et al suggested the possible existence of a third type of cysteinyl leukotriene receptor (CysLT3) in the human pulmonary artery. ${ }^{16}$

As stated above, cysteinyl leukotrienes are produced by many inflammatory cells (eosinophils, macrophages, basophils, and mast cells) and may be considered as pivotal inflammatory lipid mediators that contribute to the pathogenesis of asthma. In fact, they are able to promote eosinophil recruitment and activation, increase microvascular permeability, and cause secretion of mucus, smooth muscle constriction, and proliferation. ${ }^{17}$ Their bronchospastic effect is greater than that of histamine or methacholine in human airways, both in vivo and in vitro.

\section{Contribution of leukotriene modifiers to asthma treatment}

Based on the above-described role of leukotrienes as main inflammatory mediators, leukotriene modifiers have been developed for asthma treatment. These include CysLT1 receptor antagonists (montelukast, pranlukast, and zafirlukast), and a 5-lipoxigenase inhibitor (zileuton). These medications, although with different modes of action, result in prevention of leukotriene activity. Several clinical studies have demonstrated that leukotriene modifiers are capable of protecting inflamed airways against constriction induced by different spasmogens, reducing asthma symptoms and exacerbations, as well as the need for use of rescue bronchodilators, and decreasing the level of airway inflammation. ${ }^{18,19}$ The latter is of great importance, given that leukotriene production is not completely suppressed by treatment with corticosteroids alone. Studies have been conducted on the combined effect of leukotriene modifiers, and the efficacy of this therapeutic modality has been largely recognized.

Pranlukast was the first orally active LTRA available for the treatment of asthma, and is capable of blunting the bronchoconstriction induced by leukotriene $\mathrm{D}_{4}$. In a multicenter European study addressing the safety and tolerability of pranlukast, Barnes et al demonstrated the efficacy of the LTRA in patients with chronic asthma for the first time, as well as its safety profile. ${ }^{18}$ In 2003, Horiguchi et $\mathrm{al}^{20}$ assessed the efficacy of long-term administration of pranlukast in adult asthmatics who were poorly controlled in spite of treatment with an inhaled corticosteroid. Their study showed 
significant improvement in lung function and asthma control, accompanied by a decrease in the peripheral eosinophil count and serum eosinophil cationic protein levels, suggesting that pranlukast further reduces the degree of airway inflammation by specifically acting on eosinophils. One of the conclusions from these studies was that the antiinflammatory effects of inhaled corticosteroids and LTRAs may be complementary.

Randomized controlled trials have confirmed the efficacy of LTRAs when used as monotherapy or added to inhaled corticosteroids for improving asthma outcomes, including lung function, symptoms, asthma exacerbations, healthrelated quality of life, rates of hospitalization, and death due to asthma attacks. ${ }^{21-24}$ In a recent study, Yasui et $\mathrm{al}^{25}$ showed that addition of oral pranlukast to an inhaled corticosteroid or an inhaled corticosteroid plus a long-acting beta- 2 adrenergic agent in stable asthmatics provided additional clinical benefit. Interestingly, add-on oral pranlukast was found to significantly reduce the levels of alveolar nitric oxide, a parameter of small airway inflammation, suggesting that the additional clinical effect of oral pranlukast could be mediated by reduction in peripheral airway inflammation.

Several clinical studies have confirmed the effectiveness of montelukast in adults with both asthma and allergic rhinitis. To date, according to Global Initiative for Asthma guidelines, ${ }^{26}$ LTRAs are recommended as alternative controller medications for adults with mild persistent asthma (step 2). Moreover, patients with aspirin-sensitive asthma seem to respond well to LTRAs. ${ }^{27}$ The use of LTRAs as a steroid-sparing treatment is also encouraged in clinical practice. When used as add-on therapy, LTRAs may reduce the dose of inhaled corticosteroids required by patients with moderate to severe asthma and may improve asthma control in individuals whose asthma is not controlled with a low dose or high dose of inhaled corticosteroids.

Finally, because current treatments for acute asthma provide inadequate benefit for some patients, the efficacy of intravenous montelukast as adjunctive therapy for acute asthma was evaluated. In this regard, addition of intravenous montelukast to standard care produced a more rapid improvement in forced expiratory volume in one second $\left(\mathrm{FEV}_{1}\right)$ in acute asthma. ${ }^{28}$ More recently, the acute effect of montelukast was investigated by the same group ${ }^{29}$ in 600 adult asthmatics with $\mathrm{FEV}_{1}<50 \%$ predicted, randomly allocated to intravenous montelukast $7 \mathrm{mg}$ or placebo in addition to standard care. This randomized, double-blind, placebo-controlled study showed that intravenous montelukast, when added to standard therapy, provided significant, rapid, and sustained benefit in acute asthma. Montelukast was generally well tolerated, and its safety profile was comparable with that of placebo.

\section{Leukotriene modifiers in the elderly}

Inhaled corticosteroids are recommended in many clinical practice guidelines for asthma management ${ }^{26}$ as first-line anti-inflammatory control agents, whereas LTRAs are second-choice drugs. These guidelines do not include specific sections for the management of the disease in the elderly, thus implying that drugs indicated for asthma treatment are effective across the age groups, as suggested by the National Asthma Education and Prevention Program Working Group Report on asthma in the elderly. ${ }^{30}$ However, these age-specific guidelines were published in 1996 and never updated. It should be emphasized that advanced age is one of the main exclusion criteria for randomized controlled trials involving asthmatics. It is logical to assume that, in real-life settings, elderly asthmatic subjects are currently treated by protocols based on results of randomized controlled trials for which they would have been ineligible.

It is known that the real clinical benefit provided by inhaled therapy can be influenced by several factors, mostly related to the patient rather than to the medication itself. Amongst these, adherence plays a major role, particularly in elderly patients. In elderly patients, unintentional nonadherence with inhalation therapy represents a complex problem that may lead to significant impairment of asthma symptom control. ${ }^{31-33}$ Elderly patients often suffer from multiple chronic diseases, such as cognitive impairment, hearing or visual problems, or other physical disabilities (arthritis, tremor, and low coordination) that significantly affect their ability to inhale the drug properly. In this context, the simpler route of administration of LTRAs, compared with the inhaled agents, could provide a valid strategic option in asthma therapy.

Among the products derived from the lipoxygenase pathway of arachidonic acid metabolism, cysteinyl leukotrienes have proinflammatory functions, whereas lipoxin carry potent anti-inflammatory signals. ${ }^{34}$ Lipoxins, especially $\mathrm{LXA}_{4}$, antagonize a number of proinflammatory mediators, resulting in inhibition of leukocyte-dependent inflammation. ${ }^{34}$ Given that aging is characterized by an increased incidence of diseases with a marked inflammatory component, Gangemi et $\mathrm{al}^{34}$ tested the hypothesis that aging is associated with impairment of resolution mechanisms by measuring urinary levels of anti-inflammatory $\mathrm{LXA}_{4}$ in healthy subjects of different ages, including centenarians. They demonstrated 
that the urinary concentrations of $\mathrm{LXA}_{4}$ decrease with age, whereas cysteinyl leukotriene concentrations increase with age. As a consequence, the ratio of $\mathrm{LXA}_{4}$ to cysteinyl leukotrienes, which was proposed as an inflammation protection index, was significantly lower in the elderly. ${ }^{34}$ These results support the hypothesis that the endogenous host defense barrier against inflammatory insults tends to become impaired with age and they are in line with observations in bronchial asthma, where it has been demonstrated that adult subjects with moderate or severe asthma had lower LXA $_{4}$ concentrations in the supernatants of induced sputum than those with mild asthma. ${ }^{35}$

Older evidence suggests that zafirlukast is able to improve pulmonary function and asthma symptoms in adolescents, adults, and the elderly. ${ }^{36}$ However, this study has several limitations, including its open-label design, lack of a control group, predominance of white female patients, and a relatively short trial duration (4 weeks). Moreover, improvements in elderly patients were less dramatic compared with the other two age groups. In the elderly group, the more severe the asthma, the greater the percentage of patients with a reduction in symptoms, whereas the inverse association occurred in adolescents. ${ }^{36}$ Of note, zafirlukast was well tolerated by patients in all age groups, with treatment-related adverse events occurring in $4.2 \%$ of adolescents, $7.4 \%$ of adults, and $8.1 \%$ of elderly patients, respectively. Elderly patients had the highest incidence of headache, abdominal pain, diarrhea, and nausea, and the lowest incidence of infection and sinusitis. ${ }^{36}$ In contrast with those results, a retrospective analysis ${ }^{37}$ of five published clinical trials, in which fluticasone was compared with zafirlukast, showed that treatment with zafirlukast in asthmatic patients aged 50 years and over resulted in small improvements in asthma symptoms, minimal or no improvement in lung function, and increased exacerbations. The authors were concerned that zafirlukast could mask the deterioration of asthma in this aged population. With regard to the safety profile, it is reassuring that adverse events (headache, nausea, diarrhea, and heartburn) occurred in less than $2 \%$ of the older patients, similar to what is observed in younger asthmatics. ${ }^{37}$ The strength of this review is its evaluation of studies with a randomized and double-blind design. Taken together, these observations suggest caution in using zafirlukast in adults aged 50 years and over.

A possible explanation for these negative results could be provided by a study that aimed at characterizing asthma at a cellular and molecular level ${ }^{38}$ by comparing production of proinflammatory lipid mediators, ie, $\mathrm{LTB}_{4}$ and cysteinyl leukotrienes, both in vitro and in vivo in young and older asthmatics. In this pilot study, adult/elderly subjects (aged 50-70 years) with mild to moderate asthma showed lower levels of $\mathrm{LTB}_{4}$ and cysteinyl leukotrienes in induced sputum, with no difference in eosinophil or neutrophil counts in the airways. Moreover, in the older asthmatics, lipopolysaccharide stimulation of peripheral blood mononuclear cells resulted in less production of granulocyte macrophage colony-stimulating factor (GM-CSF), which in turn is a stimulus to produce leukotrienes. Therefore, the authors ${ }^{38}$ proposed that a potential explanation for the age-related differences in sputum leukotriene levels could be less GM-CSF in the airways of older asthmatics. The clinical implication of these results is that the lower levels of cysteinyl leukotrienes in the airways of older asthmatics may have an impact on the effectiveness of the therapy with LTRAs. These results appear to be in contrast with those of Gangemi et al, that have been previously discussed. ${ }^{34}$ However, the two studies are not comparable because of their different study populations. Indeed, Nyenhuis et $\mathrm{al}^{38}$ incorporated a very small number of "elderly" asthmatics, whereas Gangemi et al studied healthy subjects and included centenarians. In this respect, they found the apparent paradox of elevated inflammatory indices in extreme longevity, since centenarians displayed the lowest $\mathrm{LXA}_{4}$ levels and $\mathrm{LXA}_{4}$ to cysteinyl leukotriene ratio. This observation suggests that additional factors may compensate for the fall in anti-inflammatory mediators to prevent development of disease in extreme longevity.

Compliance with medication was found to be higher in patients taking pranlukast than in those taking inhaled fluticasone (about 93\% versus 81\%), ${ }^{39}$ and both drugs achieved similar reductions in the use of rescue medications, serum and sputum concentrations of eosinophil cationic protein, and similar improvements in lung function, as assessed by $\mathrm{FEV}_{1}$, compared with baseline values after one and 4 weeks. A randomized crossover trial ${ }^{25}$ with an open-label and uncontrolled design in a small number of controlled asthmatics of median age 66 (range 24-86) years showed that additional administration of oral pranlukast to stable asthmatics on inhaled therapy (corticosteroids alone or in combination with long-acting beta-adrenergic agents) had clinical benefit in terms of asthma symptoms, lung function, and inflammatory markers of proximal and peripheral airways. Since only half of this small sample included elderly patients, the use of pranlukast in elderly patients with controlled asthma requires caution.

In a multicenter Spanish study, 639 patients aged 18-79 years were randomized to receive montelukast $10 \mathrm{mg}$ $(n=326)$ or placebo $(n=313)$ once daily for 16 weeks, on 
top of regular treatment with budesonide $400-1,600 \mu \mathrm{g} /$ day throughout the study. ${ }^{21}$ Treatment with montelukast produced a significant reduction of $35 \%$ in the percentage of asthma exacerbation days compared with placebo, with a similar incidence of adverse events in both groups. No post hoc analysis was performed to evaluate the influence of age. Therefore, we can only speculate that age per se did not have any relevant impact on the outcomes. In this respect, several studies ${ }^{40-43}$ of montelukast share similar characteristics in terms of age and data analysis, ie, inclusion of patients over 65 years of age is generally limited to the smallest proportion of the sample, and no age-specific subanalysis is performed. Therefore, to establish whether older patients show similar responses compared with younger patients in terms of the efficacy and safety of montelukast may be a difficult task. This severely limits the ability to draw definite conclusions on the implementation of montelukast in elderly asthmatics.

In a double-blind, placebo-controlled, crossover, randomized add-on study, montelukast $10 \mathrm{mg}$ was added for 14 days in a total of 72 adult asthmatics (age range 22-79 years), who were symptomatic despite treatment with inhaled corticosteroids. ${ }^{44}$ In this study, the additional treatment in an outpatient setting did not result in improvement of lung function or symptom control. A more recent study ${ }^{22}$ comparing the efficacy of montelukast as an add-on therapy to inhaled corticosteroids in elderly patients with severe asthma to improve symptom control showed that montelukast plus inhaled corticosteroids for 24 months increased days without asthma symptoms, reduced days with beta-agonist use, and improved the mean asthma control test score. The authors suggested that the greater adherence to therapy observed with montelukast as compared with inhaled therapy may have contributed to the observed improvements. These results were novel, since no similar observations in a homogenous group of severe asthmatics over 60 years of age had ever been performed.

With the aim of assessing the effectiveness and tolerability of montelukast in the treatment of asthma in everyday clinical practice in representative patient groups, a retrospective cross-sectional survey of routinely collected clinical information was undertaken in 56 centers throughout the United Kingdom (20 general practice centers and 36 hospitals). ${ }^{23}$ A total of 1,429 asthmatic patients on regular treatment with montelukast were included in the analysis. Subjects ranged in age from one to 88 years, making it possible to extrapolate data for older subjects. Overall, montelukast was demonstrated to be effective across all age groups, and adverse events possibly related to montelukast occurred in a proportion similar to those seen in the montelukast and placebo groups in randomized controlled trials. However, logistic regression analysis showed a significant association between age and efficacy, meaning that an older individual was less likely to be a responder. The efficacy and safety of add-on montelukast to existing controller medications were also assessed in a real-life setting ${ }^{45}$ in a total of 5,855 individuals with asthma, whose age range was 16-96 years. Following addition of $10 \mathrm{mg}$ montelukast, the vast majority of patients reported a strong or marked improvement in day-time and night-time asthma symptoms. Similar improvement was detected for all nasal symptoms. Use of asthma and rhinitis medication was also reduced. However, a subgroup analysis specific for the elderly was not performed. Overall, montelukast was safe and well tolerated by all patients. We can infer that the evidence of a beneficial effect for montelukast in elderly asthmatics is not sufficient to suggest its use as a first-line option. However, its safety profile encourages the use of montelukast in the context of reducing $t$ use of corticosteroids, which may have detrimental effects when taken regularly for long periods by elderly asthmatics who suffer from comorbid conditions.

No clinical trial including subjects at advanced ages shows any age-related adverse effect. This reinforces the use of LTRAs at all ages, with no age restrictions. However, it is worth noting that cases of severe adverse events, such as acute hepatitis ${ }^{46}$ or Churg-Strauss syndrome, ${ }^{47}$ have been described even after a latency of years. ${ }^{46}$ Whether the elderly are more prone to adverse events, as suggested by Russmann et $\mathrm{al}^{46}$ and by Tuggey et $\mathrm{al},{ }^{47}$ opened new perspectives with suggested new indications.

In the last few years, experimental studies have opened new perspective for the LTRAs, since it has been shown that these drugs inhibit development of atherosclerosis, reduce intimal hyperplasia after vascular injury, and exert protective effects after cerebral ischemia and reperfusion. ${ }^{48}$ These observations suggest that LTRAs could potentially inhibit the inflammatory response associated with atherosclerosis, beyond their actions in asthma. On this basis, Ingelsson et $\mathrm{al}^{49}$ evaluated the effects of montelukast on the incidence and recurrence of either myocardial infarction or ischemic stroke. The results demonstrated, in a large sample of patients, that montelukast was associated with a lower risk of recurrent myocardial infarction in males and it use was associated with a lower risk of recurrent stroke in both genders. ${ }^{49}$ This provides evidence for the protective effects of montelukast 
in secondary prevention. These results could suggest a new indication for LTRAs in elderly asthmatics, given that cardiovascular disease has a higher prevalence in this age group. Indeed, if these data can be confirmed by randomized controlled trials, the LTRAs could become first-line drugs for elderly asthmatics with a history of cardiovascular events.

Recently, LTRAs have been proposed for use in elderly individuals with asthma which has become difficult to treat because of steroid resistance..$^{50}$ This could be an interesting field for use of LTRAs in the elderly, who are particularly at risk of severe asthma. However, a study conducted in 2005 by Jayaram et $\mathrm{al}^{51}$ failed to show an additive effect of montelukast over placebo for reducing sputum eosinophilia in 14 asthmatics (mean age $61.1 \pm 10.2$ years) with relative corticosteroid resistance.

In conclusion, the data available concerning the role of montelukast in the treatment of elderly asthmatics do not appear to indicate a specific role for this drug in this age group. Studies testing montelukast in the elderly population are very few $\mathrm{f}^{22,36-39}$ and have several limitations. Figure 1 summarizes the main limitations as well as the favorable aspects of using LTRAs in the elderly. Antileukotrienes may have advantages over inhaled long-acting beta-2 agonists as additive treatment with corticosteroids, given that long-acting beta-2 agonists are often contraindicated in older populations due to comorbidity, such as cardiovascular disease. Another important advantage, which is amplified in older populations, is the opportunity to achieve better adherence with oral medication. This has been demonstrated not to be a trivial issue in older patients with chronic disease. Further, the safety profile of montelukast is reassuring for physicians considering use of this drug in elderly patients. However, as indicated in Figure 1, some of the positive evidence for the effect of montelukast in the elderly appears to come from open-label studies, ${ }^{36,39}$ whereas double-blind, placebo-controlled studies show little or no effect, or were not superior to inhaled corticosteroids or combination inhaled treatments. ${ }^{44}$

Taken together, these observations are reassuring regarding safe use of montelukast in the management of asthma at advanced ages, as an add-on treatment to inhaled combination therapy to control the disease, or as an alternative to inhaled corticosteroids or long-acting beta- 2 agonists when these are contraindicated in the elderly population. Specifically designed studies to test the antiinflammatory role of montelukast in diseases other than asthma are advocated in elderly asthmatics suffering from comorbid conditions.

\section{Role of LTRAs in elderly asthmatics}

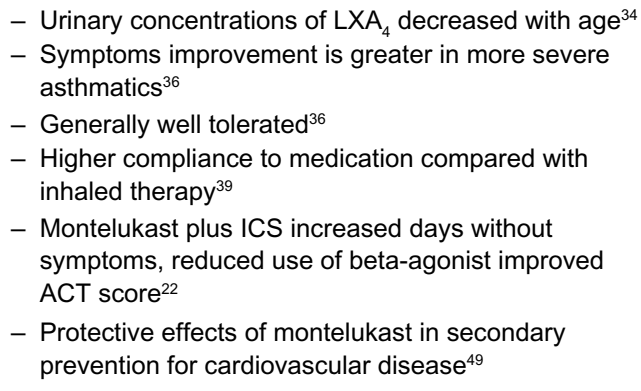

- Very few studies ${ }^{22,36-39}$ specifically designed for the elderly

- Some studies in favor of montelukast are openlabel ${ }^{36,39}$

- Studies against montelukast are double-blind, placebo-controlled studies ${ }^{44}$

- Less dramatic improvement in pulmonary function compared to adolescent and adult ${ }^{36,37}$

- Lower levels of CysLT in the induced sputum, probably due to less production of GM-CSF ${ }^{38}$

- Case reports of severe adverse events in elderly ${ }^{46,47}$

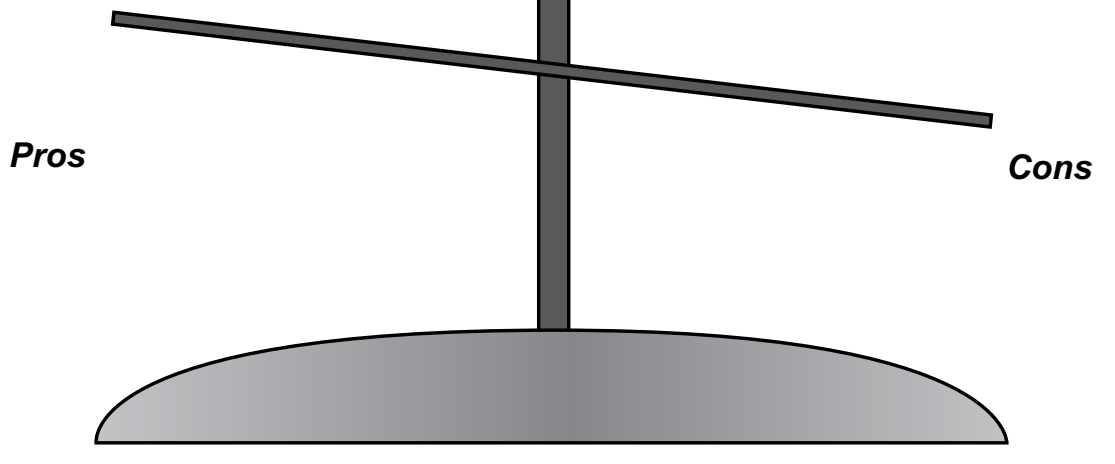

Figure I Description of the main advantages and limitations of using leukotriene modifiers in elderly asthmatics.

Abbreviations: ICS, inhaled corticosteroids; GM-CSF, granulocyte macrophage colony-stimulating factor; CysLT, cysteinyl leukotrienes; LTRAs, leukotriene antagonists; $\mathrm{ACT}$, asthma control test; $\mathrm{LXA}_{4}$, lipoxins. 


\section{Disclosure}

The authors report no conflicts of interest in this work.

\section{References}

1. Zureik M, Orehek J. Diagnosis and severity of asthma in the elderly: results of a large survey in 1,485 asthmatics recruited by lung specialists. Respiration. 2002;69(3):223-228.

2. World Health Organization. Ageing and life course. 2013. Available from: http://www.who.int/ageing/en/. Accessed July 25, 2013.

3. Bellia V, Pedone C, Catalano F, et al. Asthma in the elderly: mortality rate and associated risk factors for mortality. Chest. 2007;132(4): $1175-1182$.

4. Braman SS, Kaemmerlen JT, Davis SM. Asthma in the elderly. A comparison between patients with recently acquired and long-standing disease. Am Rev Respir Dis. 1991;143(2):336-340.

5. Connolly MJ, Crowley JJ, Charan NB, Nielson CP, Vestal RE. Reduced subjective awareness of bronchoconstriction provoked by methacholine in elderly asthmatic and normal subjects as measured on a simple awareness scale. Thorax. 1992;47(6):410-413.

6. Cuttitta G, Cibella F, Bellia V, et al. Changes in FVC during methacholineinduced bronchoconstriction in elderly patients with asthma: bronchial hyperresponsiveness and aging. Chest. 2001;119(6):1685-1690.

7. Ekici M, Apan A, Ekici A, Erdemoglu AK. Perception of bronchoconstriction in elderly asthmatics. J Asthma. 2001;38(8):691-696.

8. Law M, Morris JK, Wald N, Luczynska C, Burney P. Changes in atopy over a quarter of a century, based on cross sectional data at three time periods. BMJ. 2005;330(7501):1187-1188.

9. Linneberg A, Gislum M, Johansen N, Husemoen LL, Jorgensen T. Temporal trends of aeroallergen sensitization over twenty-five years. Clin Exp Allergy. 2007;37(8):1137-1142.

10. Scichilone N, Augugliaro G, Togias A, Bellia V. Should atopy be assessed in elderly patients with respiratory symptoms suggestive of asthma? Expert Rev Respir Med. 2010;4(5):585-591.

11. Mathur SK, Schwantes EA, Jarjour NN, Busse WW. Age-related changes in eosinophil function in human subjects. Chest. 2008;133(2): 412-419.

12. Fabbri LM, Romagnoli M, Corbetta L, et al. Differences in airway inflammation in patients with fixed airflow obstruction due to asthma or chronic obstructive pulmonary disease. Am J Respir Crit Care Med. 2003;167(3):418-424.

13. ten Brinke A, Zwinderman AH, Sterk PJ, Rabe KF, Bel EH. Factors associated with persistent airflow limitation in severe asthma. Am J Respir Crit Care Med. 2001;164(5):744-748.

14. Di Lorenzo G, Mansueto P, Ditta V, et al. Similarity and differences in elderly patients with fixed airflow obstruction by asthma and by chronic obstructive pulmonary disease. Respir Med. 2008;102(2):232-238.

15. Grayson MH, Korenblat PE. Treating asthma in the older patient: is there a place for leukotriene modifiers? Drugs Aging. 2006;23(6):451-459.

16. Back M, Kumlin M, Cotgreave IA, Dahlen SE. An alternative pathway for metabolism of leukotriene $\mathrm{D}(4)$ : effects on contractions to cysteinylleukotrienes in the guinea-pig trachea. Br J Pharmacol. 2001;133(7): 1134-1144.

17. Mechiche H, Naline E, Candenas L, et al. Effects of cysteinyl leukotrienes in small human bronchus and antagonist activity of montelukast and its metabolites. Clin Exp Allergy. 2003;33(7):887-894.

18. Barnes NC, Pujet JC. Pranlukast, a novel leukotriene receptor antagonist: results of the first European, placebo controlled, multicentre clinical study in asthma. Thorax. 1997;52(6):523-527.

19. Barnes NC, Miller CJ. Effect of leukotriene receptor antagonist therapy on the risk of asthma exacerbations in patients with mild to moderate asthma: an integrated analysis of zafirlukast trials. Thorax. 2000;55(6):478-483.

20. Horiguchi T, Tachikawa S, Kondo R, Shiga M, Hirose M, Ito T. Clinical effects of long-term administration of pranlukast, a leukotriene receptor antagonist, on adult patients with bronchial asthma. Arzneimittelforschung. 2003;53(10):714-721.
21. Vaquerizo MJ, Casan P, Castillo J, et al. Effect of montelukast added to inhaled budesonide on control of mild to moderate asthma. Thorax. 2003;58(3):204-210.

22. Bozek A, Warkocka-Szoltysek B, Filipowska-Gronska A, Jarzab J. Montelukast as an add-on therapy to inhaled corticosteroids in the treatment of severe asthma in elderly patients. J Asthma. 2012;49(5):530-534.

23. Barnes N, Thomas M, Price D, Tate H. The national montelukast survey. J Allergy Clin Immunol. 2005;115(1):47-54.

24. Ohshima N, Matsui H, Matsui Y, et al. Addition of leukotriene receptor antagonists to inhaled corticosteroids improved QOL of patients with bronchial asthma surveyed in suburban Tokyo, Japan. Allergol Int. 2011;60(4):473-481.

25. Yasui H, Fujisawa T, Inui N, et al. Impact of add-on pranlukast in stable asthma; the additive effect on peripheral airway inflammation. Respir Med. 2012;106(4):508-514.

26. Global Initiative for Asthma. Global Strategy for Asthma Management and Prevention, 2012. Available from: http://www.ginasthma.org/. Accessed August 27, 2013.

27. Dahlen SE, Malmstrom K, Nizankowska E, et al. Improvement of aspirin-intolerant asthma by montelukast, a leukotriene antagonist: a randomized, double-blind, placebo-controlled trial. Am J Respir Crit Care Med. 2002;165(1):9-14.

28. Camargo CA Jr, Smithline HA, Malice MP, Green SA, Reiss TF. A randomized controlled trial of intravenous montelukast in acute asthma. Am J Respir Crit Care Med. 2003;167(4):528-533.

29. Camargo CA Jr, Gurner DM, Smithline HA, et al. A randomized placebo-controlled study of intravenous montelukast for the treatment of acute asthma. J Allergy Clin Immunol. 2010;125(2):374-380.

30. National Asthma Education and Prevention Program. Considerations for diagnosing and managing asthma in the elderly. NIH publication 1996; No 96-3662. Available from: http://msdh.ms.gov/msdhsite/_static/ resources/2107/pdf . Accessed July 26, 2013.

31. Dolovich MB, Ahrens RC, Hess DR, et al. Device selection and outcomes of aerosol therapy: Evidence-based guidelines: American College of Chest Physicians/American College of Asthma, Allergy, and Immunology. Chest. 2005;127(1):335-371.

32. Laforest L, Denis F, Van Ganse E, et al. Correlates of adherence to respiratory drugs in COPD patients. Prim Care Respir J. 2010;19(2): $148-154$.

33. Scichilone N, Spatafora M, Battaglia S, Arrigo R, Benfante A, Bellia V. Lung penetration and patient adherence considerations in the management of asthma: role of extra-fine formulations. $J$ Asthma Allergy. 2013;6:11-21.

34. Gangemi S, Pescara L, D’Urbano E, et al. Aging is characterized by a profound reduction in anti-inflammatory lipoxin A4 levels. Exp Gerontol. 2005;40(7):612-614.

35. Vachier I, Bonnans C, Chavis C, et al. Severe asthma is associated with a loss of LX4, an endogenous anti-inflammatory compound. J Allergy Clin Immunol. 2005;115(1):55-60.

36. Korenblat PE, Kemp JP, Scherger JE, Minkwitz MC, Mezzanotte W. Effect of age on response to zafirlukast in patients with asthma in the Accolate Clinical Experience and Pharmacoepidemiology Trial (ACCEPT). Ann Allergy Asthma Immunol. 2000;84(2):217-225.

37. Creticos P, Knobil K, Edwards LD, Rickard KA, Dorinsky P. Loss of response to treatment with leukotriene receptor antagonists but not inhaled corticosteroids in patients over 50 years of age. Ann Allergy Asthma Immunol. 2002;88(4):401-409.

38. Nyenhuis SM, Schwantes EA, Mathur SK. Characterization of leukotrienes in a pilot study of older asthma subjects. Immun Ageing. 2010;7:8.

39. Horiguchi T, Tachikawa S, Kondo R, et al. Comparative evaluation of the leukotriene receptor antagonist pranlukast versus the steroid inhalant fluticasone in the therapy of aged patients with mild bronchial asthma. Arzneimittelforschung. 2007;57(2):87-91.

40. Williams B, Noonan G, Reiss TF, et al. Long-term asthma control with oral montelukast and inhaled beclomethasone for adults and children 6 years and older. Clin Exp Allergy. 2001;31(6):845-854. 
41. Rand C, Bilderback A, Schiller K, Edelman JM, Hustad CM, Zeiger RS. Adherence with montelukast or fluticasone in a long-term clinical trial: results from the mild asthma montelukast versus inhaled corticosteroid trial. J Allergy Clin Immunol. 2007;119(4):916-923.

42. Storms W, Michele TM, Knorr B, et al. Clinical safety and tolerability of montelukast, a leukotriene receptor antagonist, in controlled clinical trials in patients aged $\geq 6$ years. Clin Exp Allergy. 2001;31(1):77-87.

43. Pearlman DS, White MV, Lieberman AK, et al. Fluticasone propionate/ salmeterol combination compared with montelukast for the treatment of persistent asthma. Ann Allergy Asthma Immunol. 2002;88(2): 227-235.

44. Robinson DS, Campbell D, Barnes PJ. Addition of leukotriene antagonists to therapy in chronic persistent asthma: a randomised double-blind placebo-controlled trial. Lancet. 2001;357(9273):2007-2011.

45. Virchow JC, Bachert C. Efficacy and safety of montelukast in adults with asthma and allergic rhinitis. Respir Med. 2006;100(11):1952-1959.
46. Russmann $\mathrm{S}$, Iselin HU, Meier D, et al. Acute hepatitis associated with montelukast. J Hepatol. 2003;38(5):694-695.

47. Tuggey JM, Hosker HS. Churg-Strauss syndrome associated with montelukast therapy. Thorax. 2000;55(9):805-806.

48. Back M. Leukotriene signaling in atherosclerosis and ischemia. Cardiovasc Drugs Ther. 2009;23(1):41-48.

49. Ingelsson E, Yin L, Back M. Nationwide cohort study of the leukotriene receptor antagonist montelukast and incident or recurrent cardiovascular disease. J Allergy Clin Immunol. 2012;129(3):702-707. e702.

50. Hanania NA, King MJ, Braman SS, et al. Asthma in the elderly: Current understanding and future research needs - a report of a National Institute on Aging (NIA) workshop. J Allergy Clin Immunol. 2011; 128(Suppl 3):S4-S24.

51. Jayaram L, Duong M, Pizzichini MM, et al. Failure of montelukast to reduce sputum eosinophilia in high-dose corticosteroid-dependent asthma. Eur Respir J. 2005;25(1):41-46.
Clinical Interventions in Aging

\section{Publish your work in this journal}

Clinical Interventions in Aging is an international, peer-reviewed journal focusing on evidence-based reports on the value or lack thereof of treatments intended to prevent or delay the onset of maladaptive correlates of aging in human beings. This journal is indexed on PubMed Central, MedLine, the American Chemical Society's 'Chemical Abstracts

\section{Dovepress}

Service' (CAS), Scopus and the Elsevier Bibliographic databases. The manuscript management system is completely online and includes a very quick and fair peer-review system, which is all easy to use. Visit http://www.dovepress.com/testimonials.php to read real quotes from published authors. 